\title{
2D Direction of Arrival Estimation for Cross Array in the Presence of Mutual Coupling
}

\author{
Weiwei Hu, Aijun Zhang, and Changming Wang \\ The School of Mechanical Engineering, Nanjing University of Science and Technology, Jiangsu 210094, China \\ Correspondence should be addressed to Changming Wang; hww1986116@163.com
}

Received 6 August 2013; Accepted 12 November 2013; Published 4 March 2014

Academic Editor: Yantao Yu

Copyright ( 2014 Weiwei Hu et al. This is an open access article distributed under the Creative Commons Attribution License, which permits unrestricted use, distribution, and reproduction in any medium, provided the original work is properly cited.

\begin{abstract}
This paper proposes a new method for cross array to estimate two-dimensional direction of arrival (2-D DOA) in the presence of mutual coupling. In this method, the array elements which are affected by the same mutual coupling are chosen on $x$-axis and $z$-axis, respectively. Then a new matrix is constructed with the proper entries of cross covariance matrix of the chosen elements outputs on $x$-axis and $z$-axis. Propagation method (PM) and rotational invariance techniques for uniform linear array (ULA) are utilized in the constructed matrix to obtain two parameters correlated with elevations and azimuths. While calculating and pairing the two parameters, only once eigendecomposing and several division operations are required with the relationship among the matrix, its eigenvalues, and corresponding eigenvectors. Simulations are presented to validate the performance of the proposed method.
\end{abstract}

\section{Introduction}

Mutual coupling between elements has a serious effect on precision and resolution of the direction of arrival estimation algorithms $[1,2]$. Therefore, there are lots of works addressing this issue of how to restrain the effects of mutual coupling. Many calibration algorithms have been proposed in the past decades. Friedlander and Weiss presented an iterative procedure to compensate the mutual coupling and perturbation of gain and phase [3]. In [4], Sellone and Serra constructed an objective function based on covariance matrix matching, dealt with mutual coupling matrix and its conjugate transpose as unrelated ones, and searched the optimal solution with iterative procedure. However, the multidimensional search needed for the associated nonlinear optimal search is computationally complicated in $[3,4]$. Wang et al. made use of banded complex symmetric Toeplitz characteristic of the mutual coupling matrix of ULA and rank reduction (RARE) algorithm to obtain azimuths and mutual coupling coefficients [5], while Lin and Yang utilized banded complex symmetric circular Toeplitz characteristic of the mutual coupling matrix of uniform circle array (UCA)
[6]. These two methods are only suitable for ULA and UCA, respectively. The idea from [5] was applied to cross array, but the transformed matrix related to the ideal steering vector has no universal form for reasons of the array configuration and the variable mutual coupling degree of freedom [7]. The literature [8] presented a decoupling algorithm for ULA. It chooses the proper elements whose ideal steering vector can be separated from the mutual coupling coefficients and then employs the outputs of the chosen elements to construct MUSIC spatial spectral estimator. Nevertheless this method can only estimate one-dimensional direction and needs the spectral peak search. In $[9,10]$, this method $[8]$ was applied to uniform rectangular array (URA) and uniform hexagon array (UHA), respectively, to estimate two-dimensional directions, but the configurations of planar arrays are complicated and lots of auxiliary elements are needed.

In order to estimate 2-D DOA in the presence of mutual coupling, a method for cross array which has a simpler configuration than those for planar arrays is presented in this paper. The method is based on PM algorithm utilizing signal subspace eigenvectors algorithm for the array consisting of the chosen elements which are affected by the same mutual 


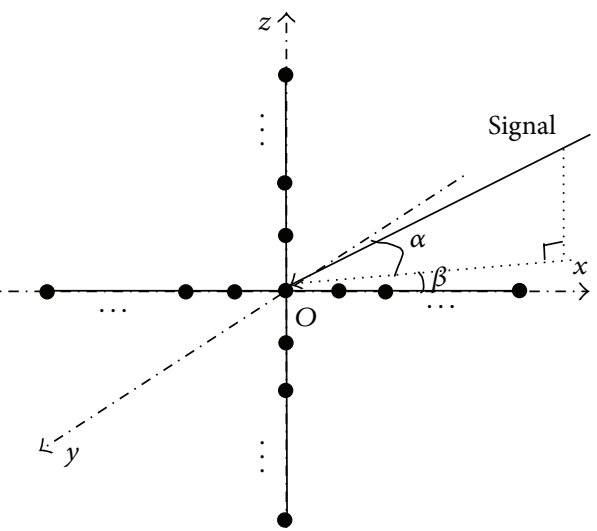

FIGURE 1: The cross array configuration.

coupling. It is not an iterative algorithm, and the spectral peak search is not required. It can estimate the 2-D DOA accurately without estimating the mutual coupling coefficients.

\section{Problem Formulation}

Consider a cross array consisting of two ULAs located on $x$-axis and $z$-axis, respectively. The ULAs share the middle element as reference at origin of coordinates. Each ULA has $2 M+1$ sensors with element spacing of $d$, which means that there exist $4 M+1$ ones in the whole array because of the sharing element. Assume that $K$ narrowband, far-field, noncoherent signals with the same wave length $\lambda(\lambda=2 d)$ impinge on this array, and the $k$ th source's elevation and azimuth are $\alpha_{k}$ and $\beta_{k}$, respectively. The array configuration is shown in Figure 1.

Let $\mathbf{C}$ and $t$ denote the mutual coupling matrix (MCM) and the time index, respectively. The output of the array can be expressed as

$$
\mathbf{Y}(t)=\mathbf{C A S}(t)+\mathbf{N}(t),
$$

where $\mathbf{Y}, \mathbf{A}, \mathbf{S}$, and $\mathbf{N}$ represent the received signal vector, the ideal steering matrix, the source signal vector, and the stochastic noise (additive white Gauss noise whose mean is zero and variance is $\sigma^{2}$ ) vector, respectively. They can be described as (the time index $t$ is omitted for brevity)

$$
\begin{gathered}
\mathbf{Y}=\left[x_{1}, \ldots, x_{M}, \ldots, x_{2 M+1}, z_{1}, \ldots, z_{M}, \ldots, z_{2 M+1}\right]^{T}, \\
\mathbf{A}=\left[\mathbf{a}\left(\alpha_{1}, \beta_{1}\right), \ldots, \mathbf{a}\left(\alpha_{k}, \beta_{k}\right), \ldots, \mathbf{a}\left(\alpha_{K}, \beta_{K}\right)\right], \\
\mathbf{a}\left(\alpha_{k}, \beta_{k}\right)=\left[e^{j 2 \pi d M \cos \alpha_{k} \cos \beta_{k} / \lambda}, \ldots, 1, \ldots,\right. \\
e^{-j 2 \pi d M \cos \alpha_{k} \cos \beta_{k} / \lambda}, \\
\left.e^{j 2 \pi d M \sin \alpha_{k} / \lambda}, \ldots, 1, \ldots, e^{-j 2 \pi d M \sin \alpha_{k} / \lambda}\right]^{T}, \\
\mathbf{S}=\left[s_{1}, \ldots, s_{k}, \ldots, s_{K}\right]^{T}, \\
\mathbf{N}=\left[n_{x_{1}}, \ldots, n_{x_{M}}, \ldots, n_{x_{2 M+1}}, n_{z_{1}}, \ldots, n_{z_{M}}, \ldots, n_{z_{2 M+1}}\right]^{T},
\end{gathered}
$$

where superscript $T$ denotes transpose.
As shown in [3], the MCM of ULA can be expressed as a banded complex symmetric Toeplitz matrix B

$$
\mathbf{B}=\text { Toeplitz }\left\{1, b_{1}, \ldots, b_{p}\right\},
$$

where integer $p$ denotes the mutual coupling degree of freedom, which means the coefficient of mutual coupling is assumed to be zero if the distance between the two sensors exceeds $p d$.

Based on the MCM model of ULA by (3), the MCM of cross array has the following form:

$$
\mathbf{C}=\left[\begin{array}{ll}
\mathbf{B} & \mathrm{D} \\
\mathbf{D} & \mathrm{B}
\end{array}\right]
$$

where $\mathbf{B}$ is the MCM of ULA on $x$ - or $z$-axis, and $\mathbf{D}$ describes the mutual coupling between the two ULAs. The matrix $\mathbf{D}$ can be presented by

$$
\mathbf{D}=\left[\begin{array}{ccc}
\mathbf{J D}_{1} \mathbf{J} & \mathbf{h}_{1}{ }^{T} & \mathbf{J D}_{1} \\
\mathbf{h}_{1} & 1 & \mathbf{h}_{1} \mathbf{J} \\
\mathbf{D}_{1} \mathbf{J} & \left(\mathbf{h}_{1} \mathbf{J}\right)^{T} & \mathbf{D}_{1}
\end{array}\right],
$$

where

$$
\begin{aligned}
& \mathbf{h}_{1}=\left[\begin{array}{llll}
\mathbf{0}_{(M-p) \times 1} & b_{p} & \cdots & b_{1}
\end{array}\right], \\
& \mathbf{J}=\left[\begin{array}{cccc}
0 & 0 & \cdots & 1 \\
0 & 0 & \cdot & \vdots \\
\vdots & 1 & \ddots & 0 \\
1 & \cdots & 0 & 0
\end{array}\right]_{M \times M}
\end{aligned}
$$

$\mathbf{D}_{1}=\left(d_{i j}\right)_{M \times M}, d_{i j}$ denotes the coefficient of mutual coupling between the two elements spacing $\sqrt{i^{2}+j^{2}} d$, and $d_{i j}$ satisfies

$$
\begin{gathered}
d_{i j}=d_{j i}, \\
d_{i j}= \begin{cases}\neq 0 & \sqrt{i^{2}+j^{2}}<p \\
=0 & \sqrt{i^{2}+j^{2}}>p .\end{cases}
\end{gathered}
$$

\section{Proposed Method}

The ideal steering vector of ULA elements affected by the same mutual coupling can be separated from the mutual coupling coefficients, which means they can be decoupled easily. Let $\tilde{\mathbf{Y}}^{\prime}$ and $\tilde{\mathbf{Y}}^{\prime \prime}$ be the outputs of elements affected by the same mutual coupling on $x$-axis and $z$-axis, respectively. 
$\widetilde{\mathbf{Y}}^{\prime}$ can be written as

$$
\begin{aligned}
\tilde{\mathbf{Y}}^{\prime} & =\mathbf{H Y} \\
& =\sum_{k=1}^{K}\left[\begin{array}{c}
b_{p} u_{k}{ }^{M}+\cdots+u_{k}^{M-p}+\cdots b_{p} u_{k}{ }^{M-2 p} \\
\vdots \\
b_{p} u_{k}^{2 p}+\cdots+u_{k}^{p}+\cdots b_{p} \\
b_{p}+\cdots+u_{k}^{-p}+\cdots b_{p} u_{k}^{-2 p} \\
\vdots \\
b_{p} u_{k}^{2 p-M}+\cdots+u_{k}^{p-M}+\cdots b_{p} u_{k}^{-M}
\end{array}\right] s_{k}+\widetilde{\mathbf{N}}_{1} \\
& =\sum_{k=1}^{K} f_{k} s_{k} \widetilde{\mathbf{a}}_{k}+\widetilde{\mathbf{N}}_{1}=\widetilde{\mathbf{A}}_{1} \mathbf{T}_{1} \mathbf{S}+\widetilde{\mathbf{N}}_{1},
\end{aligned}
$$

where $f_{k}=b_{p} u_{k}{ }^{M}+\cdots+u_{k}{ }^{M-p}+\cdots b_{p} u_{k}^{M-2 p}$,

$$
\begin{aligned}
& \mathbf{H}=\left[\begin{array}{ll}
\mathbf{H}_{1} & \underbrace{\mathbf{0}}_{2(M-2 p+1) \times(2 M+1)}
\end{array}\right], \\
& \mathbf{H}_{1}=\left[\begin{array}{ccccc}
\underbrace{\mathbf{0}}_{(M-2 p+1) \times p} & \mathbf{I} & \underbrace{\mathbf{0}}_{(M-2 p+1) \times(2 p-1)} & \mathbf{0} & \underset{(M-2 p+1) \times p}{\mathbf{0}} \\
\mathbf{0} & \mathbf{0} & \mathbf{0} & \mathbf{I} & \mathbf{0}
\end{array}\right] \text {, } \\
& \widetilde{\mathbf{a}}_{k}=\left[1, \ldots, u_{k}^{-(M-2 p)}, u_{k}^{-M}, \ldots, u_{k}^{-2 M+2 p}\right]^{T} \text {, } \\
& u_{k}=\exp \left(\frac{j 2 \pi d \cos \alpha_{k} \cos \beta_{k}}{\lambda}\right) \text {, } \\
& \widetilde{\mathbf{A}}_{1}=\left[\widetilde{\mathbf{a}}_{1}, \ldots, \widetilde{\mathbf{a}}_{k}, \ldots, \widetilde{\mathbf{a}}_{K}\right], \\
& \mathbf{T}_{1}=\operatorname{diag}\left\{f_{1}, \ldots, f_{k}, \ldots, f_{K}\right\} .
\end{aligned}
$$

There are no entries of $\mathbf{D}$ in (8) as the chosen elements on $x$-axis are not affected by the sensors on $z$-axis, because the distances between them are out of $p d$.

Similarly, $\widetilde{\mathbf{Y}}^{\prime \prime}$ can be expressed as

$$
\widetilde{\mathbf{Y}}^{\prime \prime}=\widetilde{\mathbf{A}}_{2} \mathbf{T} 2 \mathbf{S}+\widetilde{\mathbf{N}}_{2},
$$

where $\widetilde{\mathbf{A}}_{2}=\left[\widetilde{\mathbf{b}}_{1}, \ldots, \widetilde{\mathbf{b}}_{k}, \ldots, \widetilde{\mathbf{b}}_{K}\right]$,

$$
\begin{gathered}
\mathbf{T}_{2}=\operatorname{diag}\left\{g_{1}, \ldots, g_{k}, \ldots, g_{K}\right\}, \\
\widetilde{\mathbf{b}}_{k}=\left[1, \ldots, v_{k}^{-(M-2 p)}, v_{k}^{-M}, \ldots, v_{k}^{-2 M+2 p}\right]^{T}, \\
g_{k}=b_{p} v_{k}^{M}+\cdots+v_{k}^{M-p}+\cdots b_{p} v_{k}^{M-2 p}, \\
v_{k}=\exp \left(\frac{j 2 \pi d \sin \alpha_{k}}{\lambda}\right) .
\end{gathered}
$$

In order to introduce rotational invariance techniques for ULA to this array, $\tilde{\mathbf{Y}}^{\prime}$ is partitioned into two parts, so is $\widetilde{\mathbf{Y}}^{\prime \prime}$. They are denoted by $\widetilde{\mathbf{Y}}_{11}, \widetilde{\mathbf{Y}}_{12}, \widetilde{\mathbf{Y}}_{21}$, and $\widetilde{\mathbf{Y}}_{22}$, respectively:

$$
\begin{aligned}
& \tilde{\mathbf{Y}}_{11}=\left[\begin{array}{c}
\tilde{\mathbf{Y}}_{(1: M-2 p)}^{\prime} \\
\tilde{\mathbf{Y}}_{(M-2 p+2: 2 M-4 p+1)}^{\prime}
\end{array}\right], \\
& \widetilde{\mathbf{Y}}_{12}=\left[\begin{array}{c}
\tilde{\mathbf{Y}}_{(2: M-2 p+1)}^{\prime} \\
\widetilde{\mathbf{Y}}_{(M-2 p+3: 2 M-4 p+2)}^{\prime}
\end{array}\right], \\
& \widetilde{\mathbf{Y}}_{21}=\left[\begin{array}{c}
\tilde{\mathbf{Y}}_{(1: M-2 p)}^{\prime \prime} \\
\tilde{\mathbf{Y}}_{(M-2 p+2: 2 M-4 p+1)}^{\prime \prime}
\end{array}\right], \\
& \tilde{\mathbf{Y}}_{22}=\left[\begin{array}{c}
\tilde{\mathbf{Y}}_{(2: M-2 p+1)}^{\prime \prime} \\
\tilde{\mathbf{Y}}_{(M-2 p+3: 2 M-4 p+2)}^{\prime \prime}
\end{array}\right] .
\end{aligned}
$$

$\widetilde{\mathbf{Y}}_{11}$ and $\widetilde{\mathbf{Y}}_{21}$ are the submatrixes of $\widetilde{\mathbf{Y}}^{\prime}$ and $\widetilde{\mathbf{Y}}^{\prime \prime}$, respectively, so the cross covariance matrix of $\widetilde{\mathbf{Y}}_{11}$ and $\widetilde{\mathbf{Y}}_{21}$ can be built with the proper entries of cross covariance matrix of $\widetilde{\mathbf{Y}}^{\prime}$ and $\widetilde{\mathbf{Y}}^{\prime \prime}$ :

$$
\widetilde{\mathbf{R}}_{1}=\widetilde{\mathbf{A}}_{11} \widetilde{\mathbf{R}}_{s s} \widetilde{\mathbf{A}}_{21}{ }^{H}=\left[\begin{array}{ll}
\widetilde{\mathbf{R}}_{11} & \widetilde{\mathbf{R}}_{12} \\
\widetilde{\mathbf{R}}_{13} & \widetilde{\mathbf{R}}_{14}
\end{array}\right],
$$

where $\widetilde{\mathbf{A}}_{11}$ and $\widetilde{\mathbf{A}}_{21}$ are the ideal steering matrixes of arrays corresponding to $\widetilde{\mathbf{Y}}_{11}$ and $\widetilde{\mathbf{Y}}_{21}$, respectively,

$$
\begin{gathered}
\widetilde{\mathbf{R}}=E\left\{\widetilde{\mathbf{Y}}^{\prime} \widetilde{\mathbf{Y}}^{\prime \prime H}\right\}, \\
\widetilde{\mathbf{R}}_{11}=\widetilde{\mathbf{R}}_{(1: M-2 p, 1: M-2 p),} \\
\widetilde{\mathbf{R}}_{12}=\widetilde{\mathbf{R}}_{(1: M-2 p, M-2 p+2: 2 M-4 p+1)}, \\
\widetilde{\mathbf{R}}_{13}=\widetilde{\mathbf{R}}_{(M-2 p+2: 2 M-4 p+1,1: M-2 p),} \\
\widetilde{\mathbf{R}}_{14}=\widetilde{\mathbf{R}}_{(M-2 p+2: 2 M-4 p+1, M-2 p+2: 2 M-4 p+1)}, \\
\widetilde{\mathbf{R}}_{s s}=\mathbf{T}_{1} E\left\{\mathbf{S S}^{H}\right\} \mathbf{T}_{2}{ }^{H} .
\end{gathered}
$$

Similarly, let $\widetilde{\mathbf{R}}_{2}, \widetilde{\mathbf{R}}_{3}$, and $\widetilde{\mathbf{R}}_{4}$, which can be constructed with the proper entries chosen from $\widetilde{\mathbf{R}}$ as $\widetilde{\mathbf{R}}_{1}$ in (16), be the cross covariance matrixes of $\widetilde{\mathbf{Y}}_{12}$ and $\widetilde{\mathbf{Y}}_{21}, \widetilde{\mathbf{Y}}_{11}$, and $\widetilde{\mathbf{Y}}_{22}$, and $\widetilde{\mathbf{Y}}_{12}$ and $\widetilde{\mathbf{Y}}_{22}$, respectively,

$$
\begin{gathered}
\widetilde{\mathbf{R}}_{2}=\widetilde{\mathbf{A}}_{12} \widetilde{\mathbf{R}}_{s s} \widetilde{\mathbf{A}}_{21}{ }^{H}=\widetilde{\mathbf{A}}_{11} \boldsymbol{\Phi}_{1} \widetilde{\mathbf{R}}_{s s} \widetilde{\mathbf{A}}_{21}{ }^{H}, \\
\widetilde{\mathbf{R}}_{3}=\widetilde{\mathbf{A}}_{11} \widetilde{\mathbf{R}}_{s s} \widetilde{\mathbf{A}}_{22}{ }^{H}=\widetilde{\mathbf{A}}_{11} \widetilde{\mathbf{R}}_{s s}\left(\widetilde{\mathbf{A}}_{21} \boldsymbol{\Phi}_{2}\right)^{H}=\widetilde{\mathbf{A}}_{11} \boldsymbol{\Phi}_{2}{ }^{H} \widetilde{\mathbf{R}}_{s s} \widetilde{\mathbf{A}}_{21}{ }^{H}, \\
\widetilde{\mathbf{R}}_{4}=\widetilde{\mathbf{A}}_{12} \widetilde{\mathbf{R}}_{s s} \widetilde{\mathbf{A}}_{22}{ }^{H}=\widetilde{\mathbf{A}}_{11} \boldsymbol{\Phi}_{1} \boldsymbol{\Phi}_{2}{ }^{H} \widetilde{\mathbf{R}}_{s s} \widetilde{\mathbf{A}}_{21}{ }^{H},
\end{gathered}
$$

where $\widetilde{\mathbf{A}}_{12}$ and $\widetilde{\mathbf{A}}_{22}$ are the ideal steering matrixes of arrays corresponding to $\widetilde{\mathbf{Y}}_{12}$ and $\widetilde{\mathbf{Y}}_{22}$, respectively,

$$
\begin{aligned}
& \boldsymbol{\Phi}_{1}=\operatorname{diag}\left\{u_{1}^{-1}, \ldots, u_{k}^{-1}, \ldots, u_{K}^{-1}\right\}, \\
& \boldsymbol{\Phi}_{2}=\operatorname{diag}\left\{v_{1}^{-1}, \ldots, v_{k}^{-1}, \ldots, v_{K}^{-1}\right\} .
\end{aligned}
$$


The new matrix $\widetilde{\mathbf{R}}^{\prime}$ consisting of the four cross covariance matrixes includes the rotational invariance factors,

$$
\widetilde{\mathbf{R}}^{\prime}=\left[\begin{array}{c}
\widetilde{\mathbf{R}}_{1} \\
\widetilde{\mathbf{R}}_{2} \\
\widetilde{\mathbf{R}}_{3} \\
\widetilde{\mathbf{R}}_{4}
\end{array}\right]=\left[\begin{array}{c}
\widetilde{\mathbf{A}}_{11} \\
\widetilde{\mathbf{A}}_{11} \boldsymbol{\Phi}_{1} \\
\widetilde{\mathbf{A}}_{11} \boldsymbol{\Phi}_{2}{ }^{H} \\
\widetilde{\mathbf{A}}_{11} \boldsymbol{\Phi}_{1} \boldsymbol{\Phi}_{2}{ }^{H}
\end{array}\right] \widetilde{\mathbf{R}}_{s s} \widetilde{\mathbf{A}}_{21}{ }^{H} .
$$

Similar to the conventional propagator method, $\widetilde{\mathbf{R}}^{\prime}$ can be partitioned as

$$
\widetilde{\mathbf{R}}^{\prime}=\left[\begin{array}{ll}
\mathbf{G}^{T} & \mathbf{Q}^{T}
\end{array}\right]^{T},
$$

where $\mathbf{G}$ and $\mathbf{Q}$ are the first $K$ and the last $8(M-2 p)-K$ rows, respectively.

A matrix $\mathbf{P}$ (the propagator parameter) is defined to satisfy

$$
\mathbf{P}=\min _{\mathbf{P}}\|\mathbf{Q}-\mathbf{P G}\|^{2}
$$

The solution of (29) can be obtained as follows:

$$
\mathbf{P}=\mathbf{Q G}^{+}=\mathbf{Q G}^{H}\left(\mathbf{G G}^{H}\right)^{-1}
$$

With the relationship between the propagator parameter and ideal steering matrix, we obtain an equation as

$$
\mathbf{P}^{\prime}=\left[\begin{array}{l}
\mathbf{I} \\
\mathbf{P}
\end{array}\right]=\left[\begin{array}{l}
\mathbf{P}_{1} \\
\mathbf{P}_{2} \\
\mathbf{P}_{3} \\
\mathbf{P}_{4}
\end{array}\right]=\left[\begin{array}{c}
\widetilde{\mathbf{A}}_{11} \\
\widetilde{\mathbf{A}}_{11} \boldsymbol{\Phi}_{1} \\
\widetilde{\mathbf{A}}_{11} \boldsymbol{\Phi}_{2}{ }^{H} \\
\widetilde{\mathbf{A}}_{11} \boldsymbol{\Phi}_{1} \boldsymbol{\Phi}_{2}{ }^{H}
\end{array}\right] \mathbf{T}_{3},
$$

where $\mathbf{P}_{i}(i=1,2,3,4)$ is the $(2 M-4 p) \times K$ matrix and $\mathbf{T}_{3}$ is the $K \times K$ matrix with rank $K$.

Similar to ESPRIT, from (31) we can have the equations correlated with azimuths and elevations as

$$
\begin{aligned}
& \mathbf{P}_{1}^{+} \mathbf{P}_{2}=\mathbf{T}_{3}{ }^{-1} \boldsymbol{\Phi}_{1} \mathbf{T}_{3}, \\
& \mathbf{P}_{3}^{+} \mathbf{P}_{4}=\mathbf{T}_{3}{ }^{-1} \boldsymbol{\Phi}_{1} \mathbf{T}_{3}, \\
& \mathbf{P}_{1}^{+} \mathbf{P}_{3}=\mathbf{T}_{3}{ }^{-1} \boldsymbol{\Phi}_{2}{ }^{H} \mathbf{T}_{3}, \\
& \mathbf{P}_{2}{ }^{+} \mathbf{P}_{4}=\mathbf{T}_{3}{ }^{-1} \boldsymbol{\Phi}_{2}{ }^{H} \mathbf{T}_{3} .
\end{aligned}
$$

To solve the above equation sets and pair the azimuths and elevations, four times eigen-decomposing and $3[K(K+$ $1) / 2-1$ ] times calculating inner product of vectors are necessary in a general way. Now a method, which needs only once eigen-decomposing and some division operations to obtain and pair the azimuths and the elevations, is introduced with the relationship among the matrix, its eigenvalues, and corresponding eigenvectors.

First eigendecompose $\mathbf{P}_{1}{ }^{+} \mathbf{P}_{2}$, and the eigenvalues and the eigenvectors are denoted by $\lambda_{1 k}$ and $\boldsymbol{\rho}_{k}(k=1,2, \ldots, K)$, respectively Then substitute $\boldsymbol{\rho}_{k}$ into other equations, as the first one of the right equation sets in (32); the corresponding eigenvalue $\lambda_{3 k}$ can be obtained from

$$
\mathbf{P}_{1}^{+} \mathbf{P}_{3} \boldsymbol{\rho}_{k}=\lambda_{3 k} \boldsymbol{\rho}_{k} .
$$

Let $\mathbf{P}_{1}^{+} \mathbf{P}_{3} \boldsymbol{\rho}_{k}=\boldsymbol{\omega}_{k}$, and $\lambda_{3 k}$ can be expressed by

$$
\lambda_{3 k}=\frac{1}{K} \sum_{i=1}^{K} \frac{\boldsymbol{\omega}_{k i}}{\boldsymbol{\rho}_{k i}}
$$

where $\boldsymbol{\omega}_{k i}$ and $\boldsymbol{\rho}_{k i}$ are the $i$ th element of $\boldsymbol{\omega}_{k}$ and $\boldsymbol{\rho}_{k}$, respectively.

Assume that $\lambda_{2 k}$ and $\lambda_{4 k}$ denote the corresponding eigenvalues of $\mathbf{P}_{3}{ }^{+} \mathbf{P}_{4}$, and $\mathbf{P}_{2}{ }^{+} \mathbf{P}_{4}$ respectively, and they can be calculated by (34).

Using the relationship among $\lambda_{i k}(i=1,2,3,4), u_{k}, v_{k}, \alpha_{k}$, and $\beta_{k}$, the elevation $\alpha_{k}$ and the azimuth $\beta_{k}$ of the $k$ th source can be represented as

$$
\alpha_{k}=\frac{\left\{\arcsin \left\{\arg \left(\lambda_{3 k}\right) / \pi\right\}+\arcsin \left\{\arg \left(\lambda_{4 k}\right) / \pi\right\}\right\}}{2},
$$

$\beta_{k}$

$=\frac{\left\{\arccos \left\{-\arg \left(\lambda_{1 k}\right) / \pi \cos \alpha_{k}\right\}+\arccos \left\{-\arg \left(\lambda_{2 k}\right) / \pi \cos \alpha_{k}\right\}\right\}}{2}$.

Now give the computation load on the proposed method and PM algorithm with known mutual coupling. The computation load on PM algorithm with known mutual coupling is focused on the inverse of MCM, cross matrix of output of elements on $x$-axis and $z$-axis, eigen-decomposing a matrix with rank $K$, and pairing the parameters, so the computation load is $O\left((4 M+2)^{3}\right)+N(2 M+1)^{2}+O\left(4 K^{3}\right)+3 K^{2}[K(K+$ $1) / 2-1](N$ is the snapshots number); the computation load on the proposed method is focused on cross matrix of output of elements on $x$-axis and $z$-axis, once eigen-decomposing a matrix with rank $K$, and pairing the parameters, so the computation load is $N(2 M-4 p+2)^{2}+O\left(K^{3}\right)+3 K\left(K^{2}+K\right)$.

Finally, the necessary conditions for the proposed method are given as follows:

(1) for unique estimation, $2(M-2 p)>K$ is required;

(2) the sources can not be located at the blind angles which make the determinant of $\mathbf{T}_{1}$ or $\mathbf{T}_{2}$ zero.

\section{Simulation Experiments}

In this section, we carry out some representative simulation experiments to demonstrate the validity of the proposed calibration method. In all the experiments, we assume there are two uncorrelated equivalent-power sources at $\left(20^{\circ}, 10^{\circ}\right)$ and $\left(80^{\circ}, 60^{\circ}\right)$ that impinge on the cross array which consists of 53 elements $(M=13)$. The mutual coupling degree of freedom $p$ is two and the coefficients of mutual coupling are denoted by $b_{1}=0.3065+0.3951 j, b_{2}=0.1018-0.1721 j$, and $d_{11}=0.2154-0.2738 j$.

In the first experiment, the 2-D spatial spectrums of the PM algorithm with unknown mutual coupling, the PM algorithm with known mutual coupling, and the proposed method (estimate the 2-D DOAs to get the coefficients of mutual coupling with the method in [7]) are shown in Figures 2 to 4 in the condition that the signal-to-noise ration (SNR) is $5 \mathrm{~dB}$ and the number of the snapshots is 300 . 


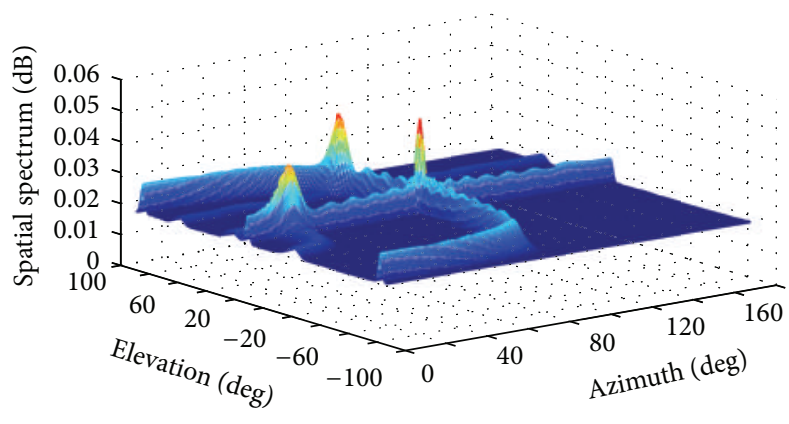

FIGURE 2: 2-D spatial spectrum of the PM algorithm in the presence of unknown mutual coupling.

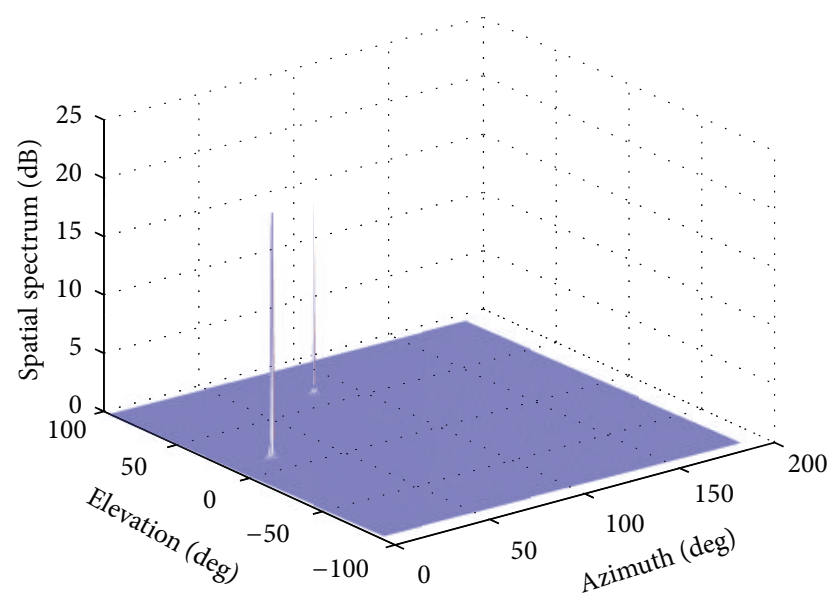

FIgURE 3: 2-D spatial spectrum of the PM algorithm in the presence of known mutual coupling.

From Figure 2 we can see that the PM algorithm without calibration has very poor resolution in the presence of mutual coupling. Though the peaks appear at the signal directions, there exist lots of pseudopeaks especially at $\left(80^{\circ}, 10^{\circ}\right)$ whose spatial spectrum is greater than the sources', which may result in the inaccurate estimation.

Comparing with Figure 4 and Figure 3, the spatial spectrum of proposed method has two sharp peaks at the signal directions, which is also shown in Figure 3. In other words, the proposed method can restrain the effects of mutual coupling and has a high resolution. Though the performance of proposed method is worse than that in Figure 3 for reasons of the array elements consumption, it eliminates pseudo peaks caused by mutual coupling, which can distinguish the two sources visibly.

In the second experiment, the effect of SNR on the performance of proposed method is explored. The number of snapshots is 300 and the SNR varies from -10 to $10 \mathrm{~dB}$ with the interval $1 \mathrm{~dB}$. The root mean square error (RMSE) which is defined as (36) is shown in Figure 5 with 100 independent experiments:

$$
\text { RMSE }=\frac{1}{2} \sum_{k=1}^{2} E\left\{\sqrt{\left(\widehat{\alpha}_{k}-\alpha_{k}\right)^{2}+\left(\widehat{\beta}_{k}-\beta_{k}\right)^{2}}\right\} .
$$

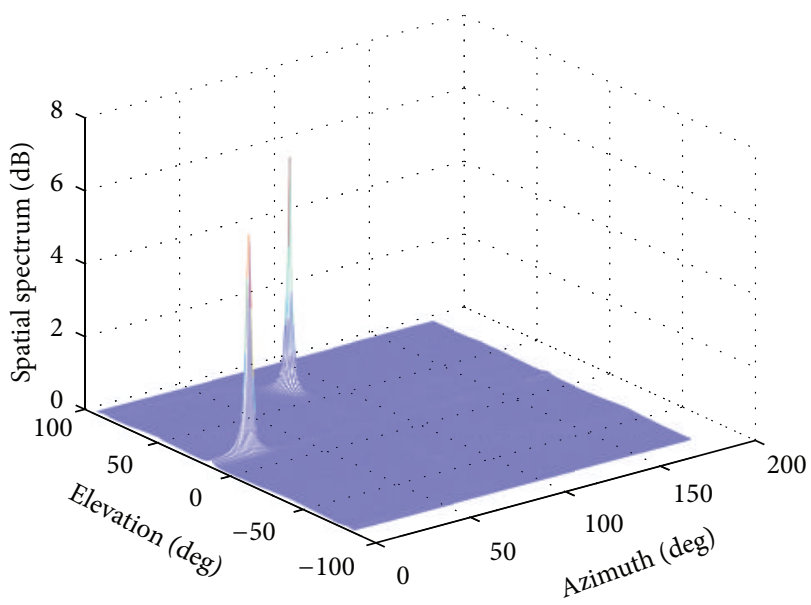

FIGURE 4: 2-D spatial spectrum of the proposed method.

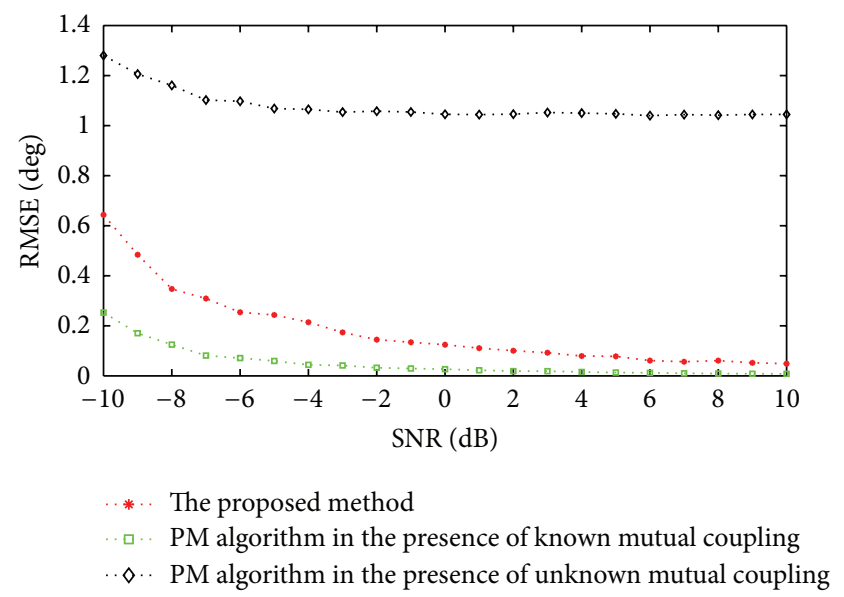

FIGURE 5: RMSE versus SNR.

The simulation results show the proposed method has an obvious performance improvement over the PM algorithm with unknown coupling, though it is a little inferior to the PM algorithm with known coupling as we think. As the SNR increases, the difference of the RMSE between the proposed method and the PM algorithm with known coupling gets closer; however, the accuracy of DOA estimates of the PM algorithm with unknown coupling stays at a low level as the main error is caused by the manifold error between the ideal and the actual one.

In the last experiment, the influence of the number of the snapshots on the performance of proposed method is investigated. SNR is $0 \mathrm{~dB}$ and the number of the snapshots varies from 100 to 800 with the interval 100. 100 independent experiments are carried out, and the RMSE curves are shown in Figure 6.

From Figure 6 we can see that the result is similar to that of the second experiment: the capability of proposed method is close to the one of the PM algorithm with known coupling, and the performance of the PM algorithm with unknown coupling is still poor when the number of the snapshots increases. 


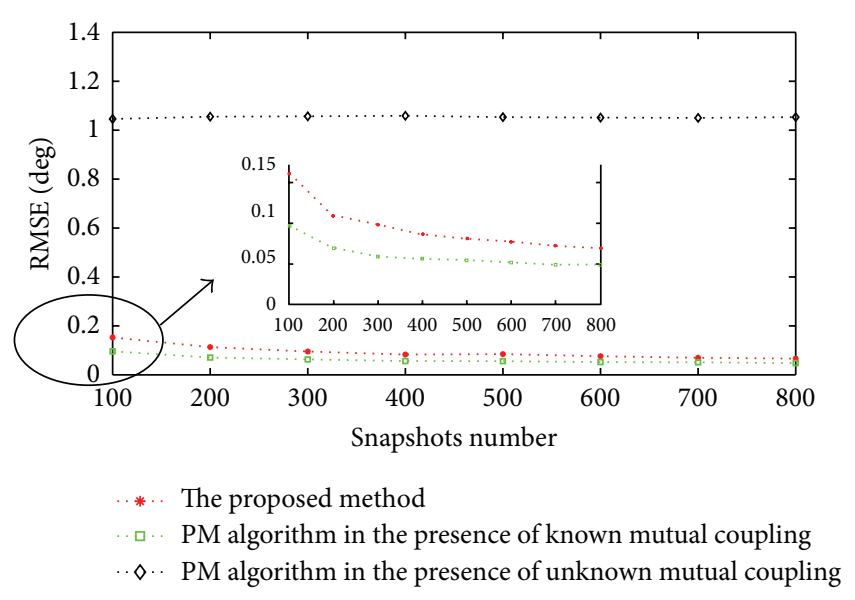

FIGURE 6: RMSE versus number of snapshots.

From the above three experiments, it is seen that the proposed method has compensated the effect caused by mutual coupling. It provides a significant improvement of DOA estimation accuracy and can achieve almost the same performance as that of PM algorithm with known mutual coupling.

\section{Conclusion}

A method compensating the effect of the mutual coupling is proposed for cross array to estimate the 2-D DOA. This method is based on PM algorithm and rotational invariance techniques for ULA. It can compensate the effect of the mutual coupling without any calibration sources, and it does not require any iterative procedure or spectral peak search. Simulations have shown that the proposed method can improve the DOA estimation accuracy well.

\section{Conflict of Interests}

The authors declare that there is no conflict of interests regarding the publication of this paper.

\section{Acknowledgments}

The authors would like to thank the editor and reviewers for their helpful comments and suggestions. In addition, this work is supported by the National High Technology Research and Development Program of China (863 Program) under Grant 2012AA0601101, the Key Laboratory of Intelligent Perception and Systems for High-Dimensional Information (Nanjing University of Science and Technology), Ministry of Education under Grant 3092013012205, and Research Fund for the Doctoral Program of Higher Education of China under Grant 20133219110027.

\section{References}

[1] C. Roller and W. Wasylkiwskyj, "Effects of mutual coupling on super resolution DF in linear arrays," in Proceedings of the
IEEE International Conference on Acoustics, Speech, and Signal Processing, vol. 5, pp. 257-260, March 1992.

[2] A. J. Weiss and B. Friedlander, "Mutual coupling effects on phase-only direction finding," IEEE Transactions on Antennas and Propagation, vol. 40, no. 5, pp. 535-541, 1992.

[3] B. Friedlander and A. J. Weiss, "Direction finding in the presence of mutual coupling," IEEE Transactions on Antennas and Propagation, vol. 39, no. 3, pp. 273-284, 1991.

[4] F. Sellone and A. Serra, "A novel online mutual coupling compensation algorithm for uniform and linear arrays," IEEE Transactions on Signal Processing, vol. 55, no. 2, pp. 560-573, 2007.

[5] B.-H. Wang, Y.-L. Wang, and H. Chen, "A robust DOA estimation algorithm for uniform linear array in the presence of mutual coupling," in Proceedings of the IEEE Antennas and Propagation Society International Symposium, vol. 3, pp. 924927, June 2003.

[6] M. Lin and L. Yang, "Blind calibration and DOA estimation with uniform circular arrays in the presence of mutual coupling," IEEE Antennas and Wireless Propagation Letters, vol. 5, no. 1, pp. 315-318, 2006.

[7] X. Hu, H. Chen, Y. Wang, and J. Chen, "A self-calibration algorithm for cross array in the presence of mutual coupling," Science China Information Sciences, vol. 54, no. 4, pp. 836-848, 2011.

[8] Z. Ye, J. Dai, X. Xu, and X. Wu, "DOA estimation for uniform linear array with mutual coupling," IEEE Transactions on Aerospace and Electronic Systems, vol. 45, no. 1, pp. 280-288, 2009.

[9] Z. Ye and C. Liu, "2-D DOA estimation in the presence of mutual coupling," IEEE Transactions on Antennas and Propagation, vol. 56, no. 10, pp. 3150-3158, 2008.

[10] C. Liu, Z. Ye, and Y. Zhang, "Autocalibration algorithm for mutual coupling of planar array," Signal Processing, vol. 90, no. 3, pp. 784-794, 2010. 

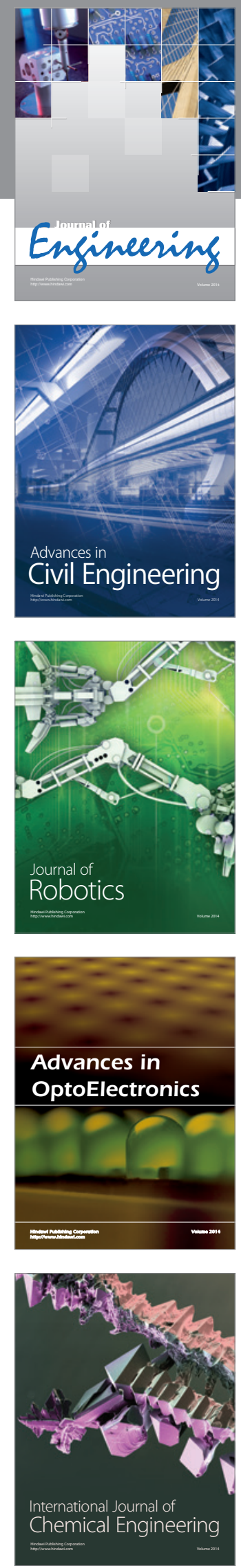

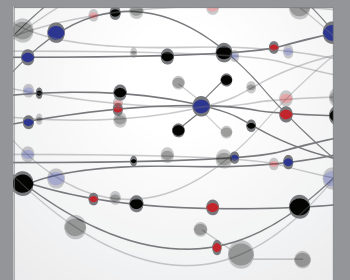

The Scientific World Journal
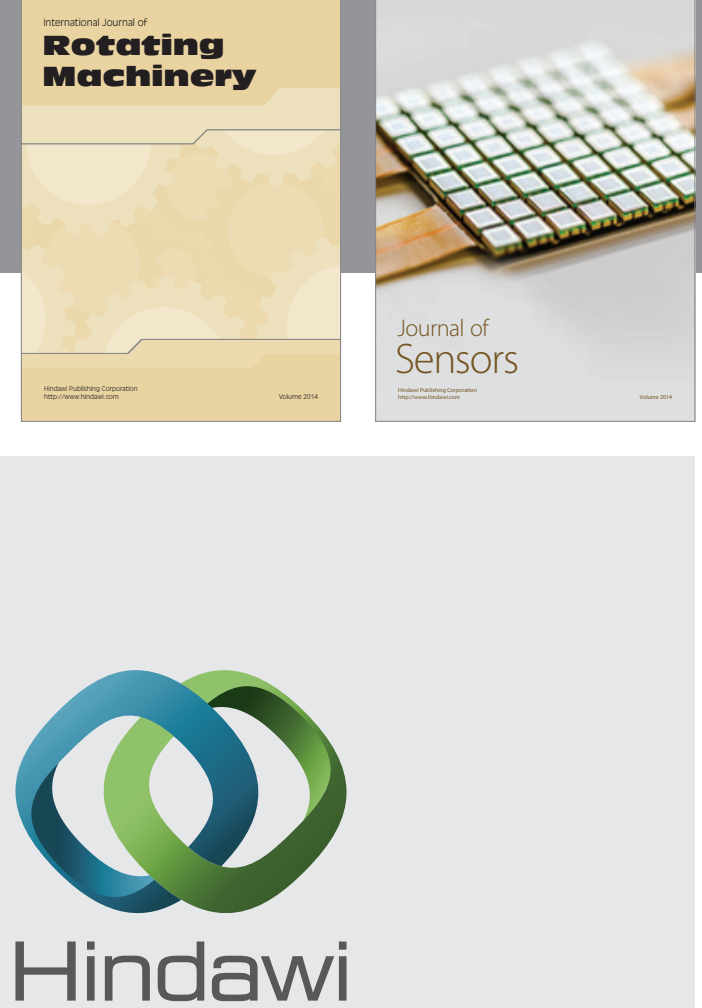

Submit your manuscripts at http://www.hindawi.com
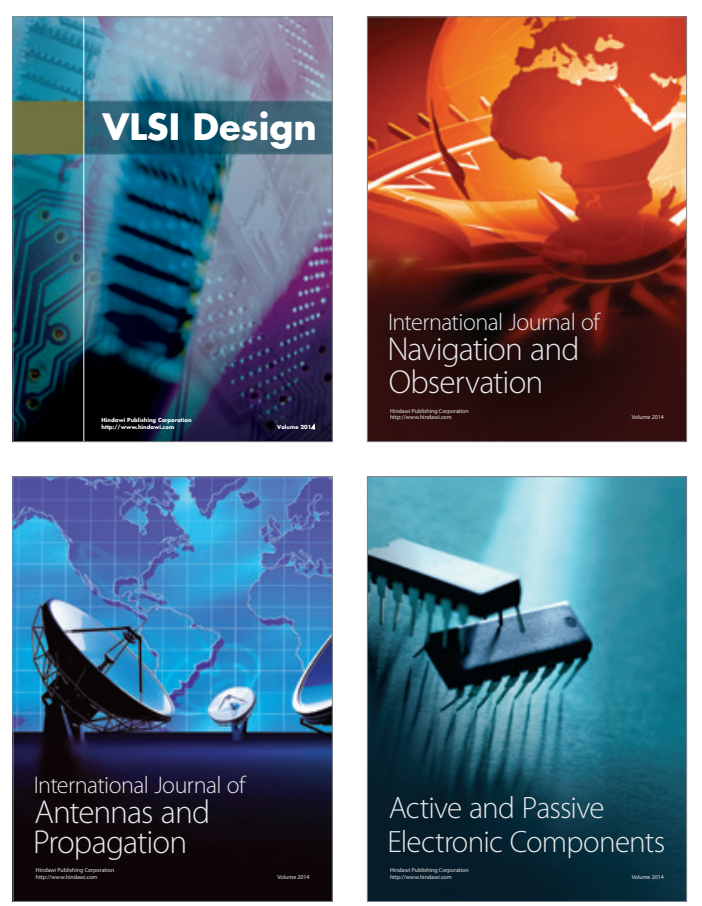
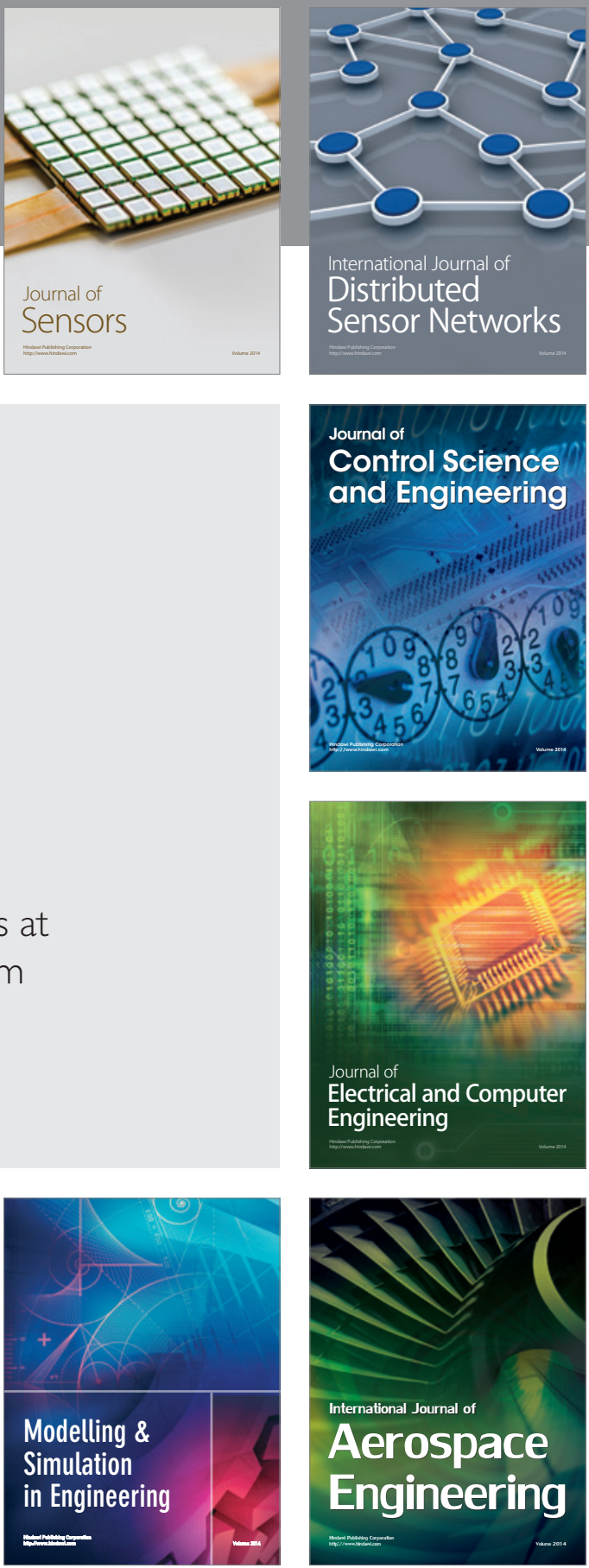

Journal of

Control Science

and Engineering
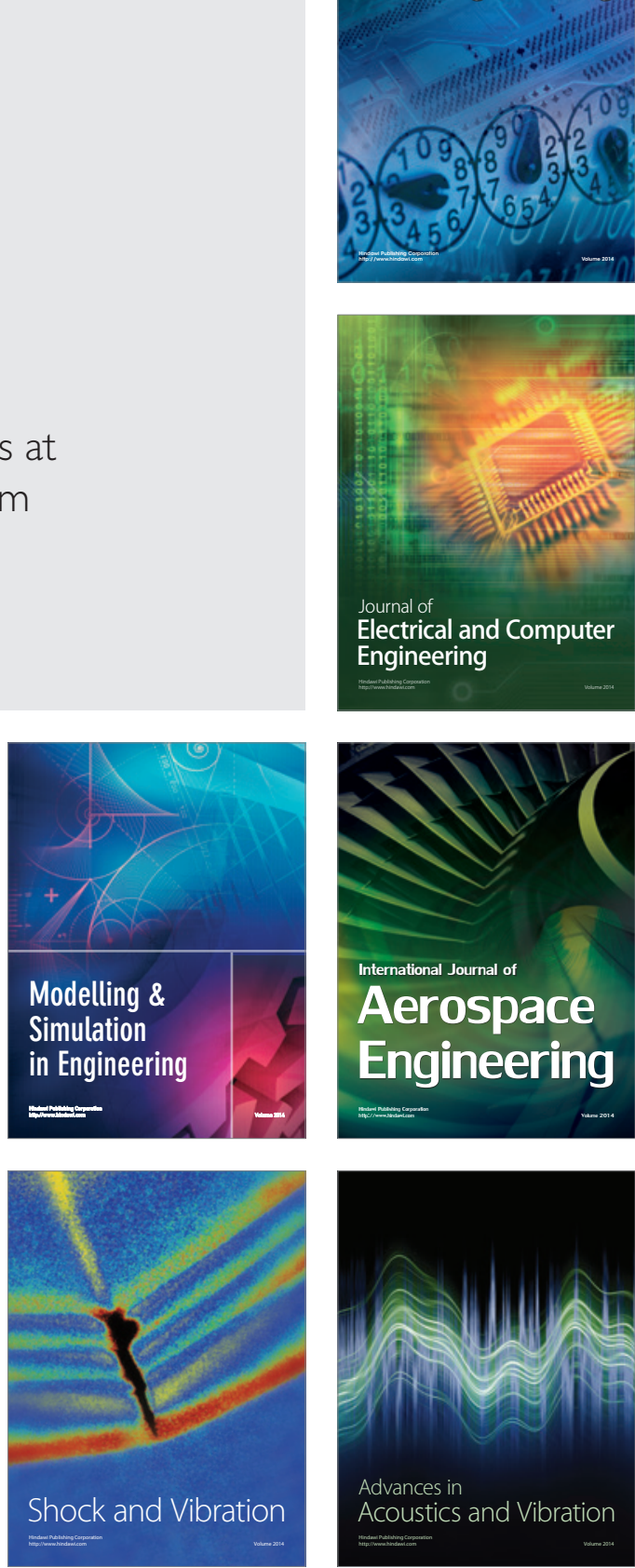